\title{
A Scorecard Method for Detecting Depression in Social Media Users
}

\author{
Netsanet Tefera Lina Zhou \\ University of Maryland, Baltimore County \\ \{netsa2,zhoul\}@umbc.edu
}

\begin{abstract}
Depression is one of the most prevalent mental health disorders today. Depression has become the leading causes of disability and premature mortality partly due to a lack of effective methods for early detection. This research explores how social media can be used as a tool to detect the level of depression in its users by proposing a scorecard method based on their user profiles. In the proposed method, depression is measured by a selected set of key dimensions along with their specific indicators, which are weighted based on their importance for signaling depression in the literature. To evaluate the scorecard method, we compared three types of social media users: users who committed suicide due to depression, users who were likely suffering from depression, and users who were unlikely suffering from depression. The empirical results demonstrate the effectiveness of the scorecard method in detecting depression.
\end{abstract}

\section{Introduction}

Depression is a pervasive and worldwide mental disorder [1,26]. Depression can become chronic or recurrent, leading to substantial impairments in an individual's ability to take care of his or her everyday responsibilities [1, 26], and even committing suicide. According to WHO's 2012 report [5], one million lives are lost yearly due to suicide, which translates into 3000 suicide deaths per day. Even though depression is treatable, the methods of identifying depression have been considered as insufficient, partly because patient's ethnicity, gender and medical conditions could jeopardize a physician's ability to correctly diagnose an individual's mental health [37]. The rise and wide adoption of social media, where people are more willing than ever to share their daily activities, feelings and emotions on one or more social media platforms (e.g., Facebook) [1, 26], has created new opportunities for detecting depression. This study investigates how to use social media in depression detection to improve users' mental health and lives.
Despite a long history research in depression, it is until very recent that researchers started to explore using social media to gain a better understanding of depression [1-3]. These studies have explored extracting information from social media posts as indicators of depression, such as the type and color of pictures posted or the textual language of posts $[1,21$, 23, 25]. However, different studies focused on different sources of information from social media, and such information remains separated rather than integrated. In addition, previous studies either fail to evaluate the overall effect of different depression indicators in detecting depression, or build machine learning models for depression detection that are difficult to use by non-expert users, primarily due to high complexity and/or low interpretability of those models. Moreover, those models typically perform binary classification without considering the level of depression.

To address the above limitations, this research introduces a novel scorecard method that integrates information from complementary sources of social media in detecting depression. In particular, the method leverages use profiles in social media as a new source of information for signaling depression. In addition to depression detection, the scorecard method has the potential to delineate the severity of depression. Furthermore, it is easy to use the scorecard method and to interpret its results.

\section{Related Work}

Two streams of research are closely related to this study: depression detection in social media, and antecedents and effects of depression.

\subsection{Depression detection in social media}

Researchers have sought to understand how people with depression share their feelings on Twitter, Instagram, and Facebook. Some researchers analyzed social media data to spot the warning signals or indicators and provide a lifeline. For instance, negative experience with using Facebook such as cyber 
bullying, meanness, misunderstandings or unwanted contacts are linked to increased depression risk among both female and male youths aged between 14 and 24 years old [11,29]. Through an analysis of 43,950 Instagram photos collected from 166 participants, one study [16] found that photos posted by depressed individuals were more likely to be grayer and darker. Other researchers analyzed the textual content of social media posts to characterize the language use. For instance, a year-long study of tweets posted by clinically depressed individuals concluded that individuals with depression show lowered social activity, greater negative emotion, higher selfattentional focus, increased relational and medicinal concerns, and heightened expression of religious thoughts [7]. These depressed individuals appeared to belong to highly clustered close-knit networks, and were typically highly embedded within their audiences based on the structure of their ego networks.

The previous studies of depression using social media are limited in that they relied on information from a single source without addressing the complexity and multi-dimensional nature of depression. In addition, their models are difficult to apply by users who do not have related technical background, because the detection results of their models are difficult to interpret and their binary results failed to recognize the severity of depression.

\section{2. Antecedents and effects of depression}

Identifying the antecedents and effects of depression is as important as detecting depression itself. Based on our review of related work (e.g., [1011, 14, 27-31], we categorized the antecedents of depression into five categories, including poverty, social culture, individual behavior, life events and demographics (see Table 1). Each of these categories further consists of a set of specific factors signaling depression. For instance, the poverty category consists of specific factors such as food insufficiency, deteriorated housing, and living in rural areas; and demographic factors include age, gender (e.g., adolescent, elderly), ethnicity (e.g., African American and White), etc. In addition, there are interaction effects between different types of demographic factors such as age and gender (e.g., adolescent female), and ethnicity, age, and gender (e.g., elderly white male). Aside from an individual's own behavior, it is also noted that life events (e.g., bullying, abuse, and death of close person) and social culture (e.g., suicide tolerance, family closeness) can contribute to depression. This review suggests that depression is a complex phenomenon that involves the interplay of a myriad of factors.
Table 1: Antecedents of depression

\begin{tabular}{|l|l|}
\hline Category & Specific factors \\
\hline Poverty & $\begin{array}{l}\text { rural areas[28], food } \\
\text { insufficiency[27], deriorated } \\
\text { housing[27] }\end{array}$ \\
\hline Social culture & $\begin{array}{l}\text { discrimination [27], suicide tolerance } \\
\text { [28], family closeness[28], stressful } \\
\text { environment [31] }\end{array}$ \\
\hline $\begin{array}{l}\text { Individual } \\
\text { behavior }\end{array}$ & $\begin{array}{l}\text { sedentary behavior [14], awkward } \\
\text { social skills[14]and } \\
\text { symptoms[10] }\end{array}$ \\
\hline Life events & $\begin{array}{l}\text { victim of any type of abuse [30], } \\
\text { bullying (particularly verbal bullying) } \\
\text { [11], death of a close person [30] }\end{array}$ \\
\hline Demographics & $\begin{array}{l}\text { adolescent female[29], African } \\
\text { white male[31] }\end{array}$ \\
\hline
\end{tabular}

The effects of suffering from depression are not limited to individual behavior, including insomnia, agitation, higher arousal than usual [34], lack of social skills [35], lack of positive affect appreciation [12], looking for similar role-models [36], lack of physical activity [15], but may also include suicidal ideation [36] and suicide of the individuals and similar behaviors of their family members [32, 33]. For instance, children of parents with major depressive disorder tend to become depressed later in their life [33]. Some of these effects overlap with the antecedents of depression. In view of the severity and broad impacts of depression, it is imperative to advance the method for detecting depression.

The detection of depression demands an integrative approach to detecting depression. The primary objective of this research is to design a method for depression detection that takes a multi-dimensional perspective in addressing the problem, and that takes ease-of-use as one of the method design considerations. The secondary research objective is to identify antecedents and effects of depression from social media and topics posted by these users.

\section{A Scorecard Method}

In this research, we introduce a scorecard method for depression detection by drawing on and extending the balanced scorecard approach [40]. The approach was originally developed to provide top managers a fast but comprehensive view of the performance of the business. Accordingly, the measures of the original 
balanced scorecard include both financial and operational measures. Creating a balanced scorecard allows managers to translate their company's strategy and mission statements into specific goals and measures. The method is found to be easy to use and effective in the organizational context, which motivates us to extend it to address of the problem of depression.

An initial step in our design of the scorecard method is to identify the goal as scoring individual social media users on their depression propensity. Accordingly, we selected measures that center on those dimensions of social media users indicative of depression. To achieve a balance among different dimensions of information, we selected measures from complementary dimensions of social media users, including: 1) timing and volume of posts, 2) color of pictures posted, 3) expressed emotion, and 4) language used. Each dimension further consists of one or more specific indicators. The indicators are selected based on both their relevance to the dimension and their public accessibility at social media sites.

Another important part of the scorecard method is the weighting scheme that signals the importance of individual dimensions and individual indicators for the detection of depression. To determine the weights, we draw on the strength of support and consistency in findings on the dimension and indicators from previous studies. To make the method easy to use, we categorized weights into three levels: low, medium, and high.

The method mainly involves creating a scorecard for a target social media user, and applying the weighting scheme to the scorecard to score his/her depression propensity. The rationales for selecting the dimensions and their indicators and for the weighting mechanism are elaborated next.

\subsection{Dimensions and Indicators of Depression}

3.1.1. Volume and timing of posts. The volume of posts can reflect how much a user feels connected to the social networks that they interact with or receive a response from. People who are believed to be suffering from depression significantly reduce their social media engagement, which is likely attributed to the decrease of a user's “social connectedness” [1]. However, only a few studies have demonstrated that the volume of social media posts can help predict depression. In addition, the level of social media engagement can vary with time and by age. For example, younger generations may feel more compelled to share information online than older generations [41]. As such, the volume of posts could vary by age. Accordingly, we set the weight of volume in signaling depression to be medium.
The number of replies to others' posts is indicative of the person's level of social interaction online. The number of original posts that are shared with others may indicate the person's desire to influence or engage others. Accordingly, we used few posts per day, few replies per day, and few links sharing within a 2-month time period, as indicators of post volume. Their weights were set to be medium, low, and low, respectively.

Depending on the timing of their posts, social media users may suffer from insomnia. Research has shown that among the people who are diagnosed with depression, 80\% experience sleep disturbance [22]. For the purpose of this study, a late night window is set to be between 11pm and 4am. We used many late night posts as an indicator of posting time, and set their weights to be medium.

3.1.2. Color of pictures posted. One study found that the colors associated with the Instagram pictures accurately determine whether a user is suffering from depression [16]. However, several studies show that there are difficulties in attributing the pictures' colors to one's emotions [23]. This is particularly true when considering the cultural background of the users [10, 23]. Thus, we set the weight of picture color for the detection of depression to be low.

Based on the color scheme, pictures can be categorized into light and dark categories. One study suggested that it was possible to determine whether someone is suffering from depression by paying attention to pictures with dark undertones [16]. The source of pictures used in the study is not limited to user profiles, but includes all of the pictures posted by the user within a chosen timeframe. Accordingly, we set the weight of dark color scheme to be medium. In addition, the study also found that depressed participants were more likely to post photos with faces than healthy participants [16]. Thus, we also considered photos with light color scheme and set its weight to be low.

3.1.3. Emotion. The type of emotion displayed can help to get a glimpse of the emotional state of a user, and possibly detect depression. Depression is associated with the inability to process emotion [1, 24, 25]. The studies suggest that studying the emotional state of a user can be a powerful tool for determining whether someone is suffering from depression. As such, we set the weight of the emotion dimension to be high in depression detection.

User emotions can be classified into two main categories: positive affect and negative affect. Positive affect refers to the state that a person might experience positive moods or emotions [25], such as being hopeful 
or positive about the future [21]. Conversely, negative emotion refers to the extent that a person experiences negative emotions or moods [25], such as having poor self-concept. We analyzed both types of user emotions in the textual and multimedia content of user posts. Depression can be associated with two types of emotional factors [1, 24, 25]: low amount of positive affects and high amount of negative affects mentioned. People who are suffering from depression are highly unlikely to be mentioning many positive affects [21, 24]. In addition, people who are not suffering from depression may still express negative affect, but likely in a much less pronounced way than people suffering from depression [25]. Thus, we set the weight of low positive affect for depression detection to be high, and that of high negative affect to be medium in depression detection.

3.1.4. Language. Research has increasingly shown that the type of language use can correctly predict depression, as it enables gauging one's emotional state more accurately [1, 21, 25]. Moreover, one study shows evidence that most depression related words are shared across cultures [18]. Thus, we set the weight for the language dimension to be high.

Table 2: The depression scorecard

\begin{tabular}{|l|l|}
\hline $\begin{array}{l}\text { Dimension } \\
\text { (weight) }\end{array}$ & Indicators (weight) \\
\hline $\begin{array}{l}\text { Volume and } \\
\text { timing of posts } \\
\text { (M) }\end{array}$ & $\begin{array}{l}\text { Few posts per day (M) } \\
\text { Few replies per day (L) } \\
\text { Few links shared per day (L) } \\
\text { Many late-night posts (M) }\end{array}$ \\
\hline $\begin{array}{l}\text { Color of } \\
\text { pictures (L) }\end{array}$ & $\begin{array}{l}\text { High light colors (L) } \\
\text { High dark colors (H) }\end{array}$ \\
\hline Emotion (H) & $\begin{array}{l}\text { Low positive affect (H) } \\
\text { High negative affect (M) }\end{array}$ \\
\hline Language (H) & $\begin{array}{l}\text { High depression related terms (H) } \\
\text { High anger related terms (H) } \\
\text { High antidepressant related terms } \\
\text { (M) }\end{array}$ \\
\hline
\end{tabular}

Note: $L, M$, and $H$ indicate low, medium, and high, respectively.

Our selection of language indicators of depression drew on two sources of information: related findings from previous studies (e.g., [1, 21, 25]), and our observations of depression-related social media posts. The latter were selected based on a list of hashtags that are indicative of depression (see Section 4.1). The hashtags consisted of three categories of terms: 1) depression-related terms such as “pain”, “alone”, “depressed”, “help”, “sad”, “suffering”, and "misunderstood"; 2) anger-related terms, which were found to be positively linked with the likelihood of someone suffering from depression [1]; and 3) antidepressant related terms, which were widely used by individuals suffering from depression in their posts, possibly seeking to receive feedback on their effects during treatment $[1,18]$. They also included vulgar and provocative terms. Based on the evidence from the literature, we set the weights of the first two categories of terms to be high and that of the last category to be medium.

Table 2 summarizes the selected measures and their weight assignments in the depression scorecard.

\subsection{Depression scoring}

In addition to the depression scorecard, applying the method also requires the selection of metrics for individual dimensions and indicators. To this end, both quantitative and quantitative methods can be used. For the sake of ease of use, the dimensions and indicators were measured on a 3-point scale: low, medium, and high, as we did with weight assignment. They were both transformed into the numerical values of 1,2, and 3 to support the scoring process.

Based on depression measures and their weights, we can determine the depression score of user $i$ $\left(\right.$ depr_score $\left._{i}\right)$ by computing the weighted average of the scorecard measures using equation (1).

$$
\begin{gathered}
\text { depr_score }_{i}=\frac{1}{\sum_{D \in\{V, C, E, L\}} w_{D}} \sum_{D \in\{V, C, E, L\}}\left(w_{D} * D_{i}\right)(1) \\
D_{i}=\frac{1}{\sum_{f \in D} w_{f}} \sum_{f \in D}\left(w_{f} * f_{i}\right),
\end{gathered}
$$

where $V, C, E$, and $L$ denote volume, color, emotion, and language dimensions, respectively; and $w_{d}$ and $w_{f}$ represent the weights assigned to dimension $D$ and indicator $f$, separately; and $D_{i}$ denotes the value of $D$ and $f_{i}$ the value $f$ for social media user $i$. The depression score ranges from 1 to 3 with 1 being least likely, and 3 most likely, suffering from depression.

\section{Evaluation}

We evaluated the proposed scorecard method by comparing the depression scores between social media users who were likely suffering from depression and users who were unlikely suffering from depression. Moreover, we also compared the depression scores between social media users who were likely suffering from depression and users who were suffering from 
severe depression. These comparisons will evaluate the performance of the scorecard method not only in detecting depression but also in detecting the severity of depression.

\subsection{A Preliminary Analysis}

To explore the feasibility of collecting depressionrelated social media data, we conducted a preliminary analysis of user posts on Facebook and Twitter. In view that both platforms support hashtags, we selected the following hashtags that have clear association with depression to retrieve related information, including \#Depressed, \#Depression, \#EffectsOfDepression, \#CausesOfDepression, and \#TalkAboutDepression. Those hashtags were measured by their post count, user count, reach, and impression. The first two measures are self-explanatory. Reach denotes the number of unique views that a post has received from other social media users, and the impression measures the potential number of total views. The descriptive statistics of the data collection is reported in Table 3.

Table 3: Descriptive statistics of sample depressionrelated hashtags in Facebook and Twitter

\begin{tabular}{|l|l|l|l|l|}
\hline \#Hashtag & $\begin{array}{l}\text { Post } \\
\text { count }\end{array}$ & $\begin{array}{l}\text { User } \\
\text { count }\end{array}$ & Reach & Impression \\
\hline Depressed & 691 & 677 & 3572703 & 3736583 \\
\hline Depression & 674 & 627 & 12096286 & 12258835 \\
\hline $\begin{array}{l}\text { EffectsOfDe } \\
\text { pression }\end{array}$ & 32 & 1 & 1 & 32 \\
\hline $\begin{array}{l}\text { CausesOfDe } \\
\text { pression }\end{array}$ & 167 & 151 & 696439 & 840005 \\
\hline $\begin{array}{l}\text { TalkAboutDe } \\
\text { pression }\end{array}$ & 117 & 108 & 361098 & 362497 \\
\hline
\end{tabular}

The results show that hashtag \#Depressed had a slightly higher frequency of use than \#Depression; however, the latter received a significantly higher number of unique views than the former. Such a discrepancy may suggest that social media users who are seeking information about depression tend to use \#Depression more, and whereas there is little difference between the choice of the two hashtags by those users who actually generated depression-related social media content. In addition, the results also reveal that social media users rarely discuss the effects of depression despite their practice importance; on the other hand, these users appear to pay much more attention to causes of depression. These observations provide additional motivations for investigating our research questions in this study.

\subsection{Data Collection and Preparation}

The overall procedure of our data selection is described in Figure 1. The first step involved selecting the data source. We selected Facebook as the platform for collecting data for three main reasons: 1) it is one of the most popular social media platforms. The enormous number of users, who share personal information in their user profiles and via interaction with other users, would increase the chance of identifying users who are suffering from depression; 2) it allows searching for private or public groups, and 3) it provides support for users to share content and express their opinions in various forms such as text (no length limit) and multimedia.

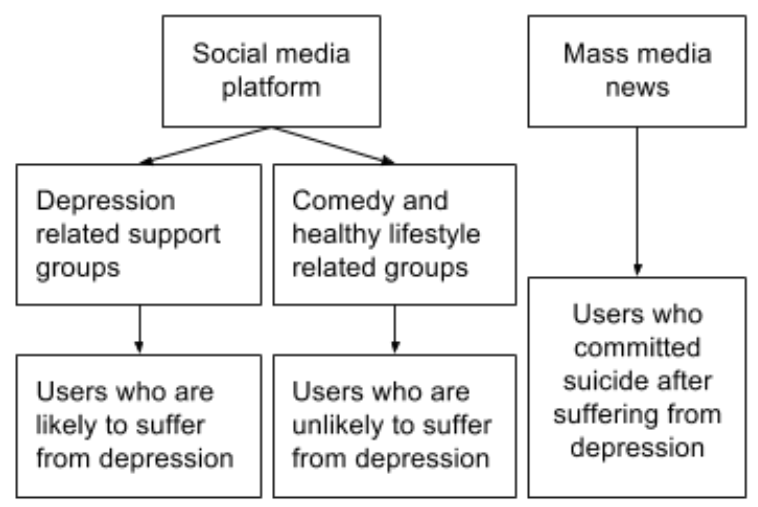

Figure 1. Data selection procedure

The second step was to identify depression-related support groups. Facebook supports both open and closed groups. In an open group, any Facebook user and non-user alike is able to view posts, but only Facebook users can actually generate posts in the group. A closed group is the opposite, where one has to make a request get approval from group moderators in order to join the group. We only considered open groups in this study for the sake of privacy protection. These Facebook support groups include: Anxiety and Depression, Help Fight Depression, Depression Group, Anxiety and Depression Support Group, Depression Support Groups, and Support Groups.

We subsequently screened users from the above depression-related support groups, particularly active members of the groups. The active members included those users who created new posts, commented on others' posts in the support groups, and/or liked other users' posts. Social media provide a rich set of data about individual users such as their user profiles, post 
content in text and/or picture, post time, and relationships. We chose to select users based on social media profiles primarily because the profiles play a fundamental role in users' disclosing their identity in a social media setting, and identity is core to many social media platforms [39]. Typically, a user profile may contain information such as name, gender, profession, and location, which serves as a way of selfpresentation. One can draw an analogy between user social media profiles and business cards and email signatures [39]. Users reveal themselves through the conscious or unconscious disclosure of subjective information such as thoughts, feelings, likes, and dislikes [40]. Thus, social media profiles are likely to reveal signs or symptoms of depression. Further, we only considered user profiles that had been made public to respect user privacy. For users with public profiles, their posts are generally available for public access regardless whether a viewer is in their "friends" list or not. Finally, we randomly selected 30 user profiles who were likely to experience depression from the active members who created new posts within a month from the time of the data collection.

The selection of users who were unlikely to experience depression followed the method of previous studies [21, 25]. Specifically, we selected the profiles of those social media users who were expected to post content with positive affect on their Facebook walls. We retrieved 12 such user profiles from comedyoriented groups and healthy lifestyle groups such as Comedy Central, Just for Laughs Gags, Healthy Lifestyle, Funny Texts, and Natural Healing.

The selection of social media users who experienced severe depression was determined based on whether the user who had depression ended up committing suicide. To this end, we started with retrieving related case reports from online mass media and manually matching those cases to their Facebook profiles. We filtered those users who were celebrities or public figures because they may avoid openly discussing about depression to protect their public images. We identified a total of 8 users who experienced severe depression.

\subsection{Data Analysis}

The data analyses started with computing the depression scores of individual social media users. Based on content analysis of the selected user profiles in social media, we scored each indicator for each user using on a 3-point scale. For example, Table 4 illustrates the scorecard of a Facebook user who was identified as being likely suffering from depression. The scores show that the user posted few messages and few links, and displayed low positive affect. In addition, the user generated a high ratio of late-night posts, dark color pictures, negative affect, and frequently used depression- and anger-related terms. The user also looked very upset, sad, and depressed in shared selfies, and almost every post had mentions of negative affect. Based on equation (1), the depression score of the user was 2.7.

Table 4: A sample scorecard

\begin{tabular}{|l|l|c|}
\hline Dimension & Indicators & Score \\
\hline Volume of & Few posts per day & 3 \\
posts & Few replies per day & 1 \\
& Few links shared per day & 3 \\
& Many late-night posts & 3 \\
\hline \multirow{2}{*}{ Color of } & High ratio of light color & 1 \\
pictures & High ratio of dark color & 3 \\
\hline \multirow{2}{*}{ Emotion } & Low positive affect & 3 \\
& High negative affect & 3 \\
\hline \multirow{2}{*}{ Language } & Frequent depression terms & 3 \\
& Frequent anger terms & 3 \\
& Frequent anti-depressant terms & 1 \\
\hline
\end{tabular}

We conducted univariate ANOVA by treating user type (LikelyD: users who were likely suffering from depression vs. SevereD: users who committed suicide after suffering from severe depression vs. NoD: users who were unlikely suffering from depression) as the independent variable, and depression score as the dependent variable. To gain an insight into the efficacy of individual dimensions of the scorecard, we also analyzed the effect of user type on each dimension separately. If the main effect of user type was detected, we followed up with post-hoc multiple comparisons with Bonferroni adjustment to evaluate the performance of the scorecard method in detecting depression and the level of depression.

\section{Results}

\subsection{Depression Scores}

Table 5 reports the descriptive statistics of individual dimensions and the overall depression scores of the three types of social media users.

The results show that mean depression score of the SevereD users $(M=2.14)$ is higher than that of LikelyD users $(M=2)$, and the latter is in turn higher than the depression score of $\mathrm{NoD}(\mathrm{M}=1.28)$. The results of univariate ANOVA yielded a significant effect of user 
type on depression score $(p<.001)$. In addition, the results of post-hoc multiple comparisons of user type show that the depression scores of both SevereD and LikelyD were higher than that of $\operatorname{NoD}(p<.001)$, but the difference between the former two types of users was not significant $(p>.05)$.

Table 5. Means [Standard deviations] of scores of three types of social media users

\begin{tabular}{|l|l|l|l|}
\hline Dimension & SevereD & LikelyD & NoD \\
\hline Volume & $2.02[.41]$ & $2.01[.45]$ & $1.25[.30]$ \\
\hline Color & $1.46[.47]$ & $1.74[.60]$ & $2.08[.73]$ \\
\hline Emotion & $2.08[.68]$ & $2.26[.66]$ & $1.1[.18]$ \\
\hline Language & $2.55[.3]$ & $1.82[.53]$ & $1.19[.27]$ \\
\hline Depr_score & $2.14[.39]$ & $2.00[.35]$ & $1.28[.14]$ \\
\hline
\end{tabular}

We repeated the above analyses for each dimension of the scorecard separately. The results show that the effect of user type was significant for all dimensions $(p<.001)$ except for color $(p<.1)$. The results of posthoc multiple comparisons of user type show that the volume scores of both LikelyD $(p<.001)$ and SevereD $(p<.01)$ were higher than that of NoD, but no difference was detected between the former two $(p>.05)$. The analyses of the emotion dimension yielded similar findings to those of volume dimension. The results of analyzing the language dimension reveal that the scores of both LikelyD and SevereD were higher than that of NoD $(p<.001)$. In addition, the language score of SevereD was higher than that of LikelyD $(p<.01)$.

The distributions of depression scores of social media users who were and were not experiencing depression allowed us to set an empirical threshold for separating them. The data suggests that users who scored below 1.3 are unlikely to experience depression, and users who scores above 2.35 are likely to experience depression.

\subsection{Antecedents and Effects of Depression}

Among the LikelyD users, 13 (43.3\%) explicitly described what have caused their depression and its effects on their personal lives. To gain a fuller understanding of such factors, we also extracted related information about suicidal role models from the mass media. We grouped these antecedents and effects of depression into six categories: work, family and relationships, victim of abuse, self-harm, traumatic events and others (e.g., disability).

- The work category concerns the stress that social media users experienced at work, their desire to stay home instead of going to work, and their unemployment status.
- $\quad$ The family and relationships category involves family-related issues such as struggling to take the responsibilities of being a parent, having depressed family members, and going through difficult breakups or divorces.

- The victim of abuse category encompasses constant physical and mental abuse, such as bullying and emotional neglect, and constant physical beating.

- The self-harm category refers to individuals who relied on unhealthy types of mediation to alleviate their depression. The most common self-induced pain involves cutting themselves, which is followed by substance abuse.

- The traumatic event category involves onetime events that had severe impact on the lives of social media users. Sample events include the death of close friends and family members, robbery, rape, and spending time in prison.

- The others category covers issues such as mental and physical disabilities.

Table 6: User mentioning distributions of antecedents and effects of depression

\begin{tabular}{|l|l|l|}
\hline Types & LikelyD & SevereD \\
\hline Work & $23.1 \%$ & $33.3 \%$ \\
\hline Family and relationships & $46.2 \%$ & $66.7 \%$ \\
\hline Victim of abuse & $15.4 \%$ & $50 \%$ \\
\hline Self-harm & $23.1 \%$ & $16.7 \%$ \\
\hline Traumatic event & $15.4 \%$ & $33.3 \%$ \\
\hline Disability & $7.7 \%$ & $0 \%$ \\
\hline
\end{tabular}

Table 6 provides a summary of both antecedents and effects of depression because it was sometimes difficult to distinguish them. For example, a user described that burglars broke into his house multiple times and how it had really affected him emotionally. Meanwhile, his posts also revealed his struggles with depression long before the burglary incident. The table reveals that family and relationships are the predominant cause of depression. In addition, 50\% of the SevereD users were victims of abuse, particularly bullying and neglect, which is much higher than that of LikelyD users (i.e., 15.4\%).

\subsection{Topics of social media post}

We identified topics by analyzing the content of social media posts generated by users who experienced depression. These topics include injustice (e.g., government injustice, minority injustice), hope/optimism (e.g., encouragement), depression (e.g., 
causes and effects of depression), despair (e.g., pleading for someone to talk to them, suicide glorification), negative perception of themselves by other people (e.g., "I know you will not like to see my legs this summer”), carelessness about others (e.g., anger-fueled posts stating they do not care about others' perception), religion (e.g., religious quotes), family pictures (e.g., family albums), pet pictures (e.g., pet albums), indoor close-up selfies (e.g., pictures that show only the face and limited amount of neck and hair), pictures with filters (e.g., Facebook filters showing support for a cause), and raising awareness about a cause (e.g., sharing or creating petitions).

Table 7: Topic distribution of social media posts

\begin{tabular}{|l|l|l|}
\hline Topic categories & LikelyD & SevereD \\
\hline injustice & $33.3 \%$ & $16.7 \%$ \\
\hline religion & $20.0 \%$ & $16.7 \%$ \\
\hline hope/optimism & $56.7 \%$ & $16.7 \%$ \\
\hline depression & $56.7 \%$ & $83.3 \%$ \\
\hline despair & $50 \%$ & $66.7 \%$ \\
\hline negative perception by others & $43.3 \%$ & $50 \%$ \\
\hline not caring about others & $33.3 \%$ & $33.3 \%$ \\
\hline family pictures & $33.3 \%$ & $50 \%$ \\
\hline pet pictures & $16.7 \%$ & $16.7 \%$ \\
\hline indoor close-up selfies & $50 \%$ & $50 \%$ \\
\hline pictures with filters & $30 \%$ & $0 \%$ \\
\hline raising awareness about a cause & $50 \%$ & $0 \%$ \\
\hline
\end{tabular}

The topic distributions of social media posts by the two types of depression users are reported in Table 7. The table shows that both types of users were likely to express despair, depression, and take very close-up indoor selfies. On the other hand, the LikelyD users posted more about hope/optimism and raising awareness about a cause than severed users, showing the former users' empathy towards others. In addition, over $40 \%$ of both types of users seemed to be highly self-conscious by posting about others' negative perception of themselves.

\section{Discussion}

\subsection{Findings}

The proposed method scored social media users who were (likely) experiencing depression significantly higher than users who did not have the problem. The findings provide strong evidence for the effectiveness of the scorecard method.

The results of this study also show that the volume, emotion, and language dimensions of social media user profiles are effective in detecting depression. Moreover, the method scored users who had severe depression higher than users who were likely suffering from depression in the language dimension. However, the color of pictures was not found to be useful.

This study identifies a wide range of antecedents and effects of depression such as family and relationships and victims of bully. Based on the topics of social media posts, users who were likely suffering from depression were more hopeful and optimistic, and more likely involving themselves in a cause than severely depressed users. These findings highlight the significant implications of (early) depression detection.

\subsection{Research Contributions}

This study makes multifold contributions to the depression research. First, this research provides a scorecard method for detecting depression in social media users. The empirical evaluation with real-world data demonstrated the effectiveness of the method. In addition, the scorecard is easy to use, and can be adapted in alignment with the strategies and goals of detection. Second, the present study integrates multiple dimensions of social media user profiles in the detection of depression. The findings not only confirmed the efficacy of some dimensions of social media users from previous studies such as volume and emotion, but also provided evidence for the role of the language dimension in determining the severity of depression. Third, the analyses of antecedents and effects of depression reveal that family and relationships are the primary factors. They suggest cultivating healthy and supportive family and relationships is promising for detecting and preventing depression. Fourth, this study uncovers topics of social media posts by users with depression, which can have implications for detecting depression such as extremely close-up selfies.

\subsection{Limitations and Future Research}

This study has limitations which motivate future research. First, the dimension design of the scorecard can be improved by incorporating the best practice of clinical psychologists. For example, users' online social network is worth to explore om depression detection, which may include factors such as the number of followers and the numbers of comments received. Second, a user's social media profile may not be up to date or consistent. For instance, users with 
happy and positive social media profiles could frequently use depression-related terms in their posts. Third, we conducted manual analysis of user profiles in social media to score individual users. The efficiency of the scoring process can be improved by employing sentiment analysis, natural language processing, and image analysis techniques.

\section{Conclusion}

Depression has become a growing concern for both families and the society at large. People could feel depressed and even have suicidal tendencies at various points of their lives. Lack of interaction with people around them or someone to hear them out often makes them vulnerable to suicidal thoughts. For users who are suffering from depression, social media emerge as an outlet of self-expressions or attempts to seek support. This research provides an effective and easy-to-use method for the detection of depression in social media users. It also improves our understanding of the antecedents and effects of depression and topics posted by depressed users. The research findings have important implications for the detection and even prevention of depression.

\section{Acknowledgements}

This research was supported in part by the National Science Foundation (SES-152768, CNS-1704800). Any opinions, findings or recommendations expressed here are those of the authors and are not necessarily those of the sponsor of this research.

\section{References}

[1] De Choudhury, M., Counts, S., \& Horvitz, E. (2013, May). Social media as a measurement tool of depression in populations. In Proceedings of the 5th Annual ACM Web Science Conference (pp. 47-56). ACM.

[2] Wang, X., Zhang, C., Ji, Y., Sun, L., Wu, L., \& Bao, Z. (2013, April). A depression detection model based on sentiment analysis in micro-blog social network. In PacificAsia Conference on Knowledge Discovery and Data Mining (pp. 201-213). Springer Berlin Heidelberg.

[3] Huijie Lin,Jia Jia,Liqiang Nie, Guangyao Shen,and TatSeng Chua 2012. What Does Social Media Say about Your Stress. Proceedings of the Twenty-Fifth International Joint Conference on Artificial Intelligence (IJCAI-16).

[4] Henner Gimpel, Christian Regal, Marco Schmidt 2015. myStress: Unobtrusive Smartphone-based Stress Detection. Proceedings of the 23th European Conference on
Information Systems (ECIS 2015), Münster, Germany, May 2015.

[5] World Health Organization, World suicide prevention day 2012. http://www. who.int/mediacentre/events/annual/world_ suicide_prevention_day/en/

[6] Veenendaal, A., Daly, E., Jones, E., Gang, Z., Vartak, S., \& Patwardhan, R. S. (2015). Sentiment Analysis in Code Review Comments. Computer Science and Emerging Research Journal, 3.

[7] Bae, Y., \& Lee, H. (2012). Sentiment analysis of Twitter audiences: Measuring the positive or negative influence of popular twitterers. Journal of the American Society for Information Science and Technology, 63(12), 2521-2535.

[8] Li, T. M., Chau, M., Wong, P. W., \& Yip, P. S. (2012, May). A hybrid system for online detection of emotional distress. In Pacific-Asia Workshop on Intelligence and Security Informatics (pp. 73-80). Springer Berlin Heidelberg.

[9] Mohammad, S. M. (2015). Sentiment analysis: Detecting valence, emotions, and other affectual states from text. Emotion Measurement, 201-238.

[10] Niemi, M., Målqvist, M., Giang, K. B., Allebeck, P., \& Falkenberg, T. (2013). A narrative review of factors influencing detection and treatment of depression in Vietnam. International journal of mental health systems, 7(1), 15.

[11] Kodish, T., Herres, J., Shearer, A., Atte, T., Fein, J., \& Diamond, G. (2016). Bullying, depression, and suicide risk in a pediatric primary care sample. Crisis.

[12] Peeters, F., Nicolson, N. A., Berkhof, J., Delespaul, P., \& deVries, M. (2003). Effects of daily events on mood states in major depressive disorder. Journal of abnormal psychology, 112(2), 203.

[13] Niederkrotenthaler, T., Arendt, F., \& Till, B. (2015). Predicting intentions to read suicide awareness stories. Crisis.

[14] Snippe, E., Simons, C. J., Hartmann, J. A., MenneLothmann, C., Kramer, I., Booij, S. H., ... \& Wichers, M. (2016). Change in daily life behaviors and depression: Within-person and between-person associations. Health Psychology, 35(5), 433.

[15] Wichers, M., Peeters, F., Rutten, B. P., Jacobs, N., Derom, C., Thiery, E., ... \& van Os, J. (2012). A time-lagged momentary assessment study on daily life physical activity and affect. Health Psychology, 31(2), 135.

[16] Reece, A. G., \& Danforth, C. M. (2017). Instagram photos reveal predictive markers of depression, EPJ Data Science, 6(1) (2017): 15. 
[17] Watson, D., Clark, L. A., \& Carey, G. (1988). Positive and negative affectivity and their relation to anxiety and depressive disorders. Journal of abnormal psychology, 97(3), 346.

[18] Ramirez-Esparza, N., Chung, C. K., Kacewicz, E., \& Pennebaker, J. W. (2008, March). The Psychology of Word Use in Depression Forums in English and in Spanish: Texting Two Text Analytic Approaches. In ICWSM.

[19] NEDARC - The National EMSC Data Analysis Resource Center, University of Utah. (n.d.). Standard Deviation. Retrieved May 20, 2017, from http://www.nedarc.org/statisticalHelp/basicStatistics/standard Deviation.html

[20] Global social media ranking 2017 | Statistic. (n.d.). Retrieved April 14, 2017, from https://www.statista.com/ statistics/272014/global-social-networks-ranked-by-numberof-users

[21] Seligman, Martin EP, and Mihaly Csikszentmihalyi. Positive psychology: An introduction. Springer Netherlands, 2014.

[22] Bower, Bethanne, et al. "Poor reported sleep quality predicts low positive affect in daily life among healthy and mood-disordered persons." Journal of sleep research 19.2 (2010): 323-332.

[23] Machajdik, Jana, and Allan Hanbury. "Affective image classification using features inspired by psychology and art theory." Proceedings of the 18th ACM international conference on Multimedia. ACM, 2010.

[24] Joormann, Jutta, and Ian H. Gotlib. "Emotion regulation in depression: relation to cognitive inhibition." Cognition and Emotion 24.2 (2010): 281-298.

[25] Miller, D. N. (2011). Positive affect. In Encyclopedia of child behavior and development (pp. 1121-1122). Springer US.

[26] Park, Minsu, David W. McDonald, and Meeyoung Cha. "Perception Differences between the Depressed and NonDepressed Users in Twitter." ICWSM. 2013.

[27] Siefert, Kristine, et al. "Modifiable risk and protective factors for depressive symptoms in low-income African American mothers." American Journal of Orthopsychiatry 77.1 (2007): 113.

[28] Park, BC Ben, Jeong Soo Im, and Kathryn Strother Ratcliff. "Rising youth suicide and the changing cultural context in South Korea." Crisis (2014).

[29] Wade, Terrance J., John Cairney, and David J. Pevalin. "Emergence of gender differences in depression during adolescence: National panel results from three countries." Journal of the American Academy of Child \& Adolescent Psychiatry 41.2 (2002): 190-198.
[30] Trice, Pamela D., and Jeffrey P. Bjorck. "Pentecostal perspectives on causes and cures of depression." Professional Psychology: Research and Practice 37.3 (2006): 283.

[31] Jensen, Hanne Voldby, Karen P. Munk, and Svend Aage Madsen. "Gendering late-life depression? The coping process in a group of elderly men." Nordic Psychology 62.2 (2010): 55.

[32] Roetker, Nicholas S., et al. "Assessment of genetic and nongenetic interactions for the prediction of depressive symptomatology: an analysis of the Wisconsin longitudinal study using machine learning algorithms." American journal of public health 103.S1 (2013): S136-S144.

[33] Marmorstein, Naomi R., and William G. Iacono. "Associations between depression and obesity in parents and their late-adolescent offspring: a community-based study." Psychosomatic medicine 78.7 (2016): 861-866.

[34] Ribeiro, Jessica D., et al. "Capability for suicide interacts with states of heightened arousal to predict death by suicide beyond the effects of depression and hopelessness." Journal of affective disorders 188 (2015): 53-59.

[35] Feldman, Jason S., Irene Tung, and Steve S. Lee. "Social Skills Mediate the Association of ADHD and Depression in Preadolescents." Journal of Psychopathology and Behavioral Assessment (2016): 1-13.

[36] Niederkrotenthaler, Thomas, Florian Arendt, and Benedikt Till. "Predicting intentions to read suicide awareness stories." Crisis (2015).

[37] Borowsky, Steven J., et al. "Who is at risk of nondetection of mental health problems in primary care?." Journal of general internal medicine 15.6 (2000): 381-388

[38] "How does Keyhole measure Reach and Impressions?" Keyhole Support. Web. 13 June 2017. <https:// keyholeco.zendesk.com/hc/en-us/articles/ 203436115-Howdoes-Keyhole-measure-Reach-and-Impressions-> .

[39] Kietzmann, J. H., Hermkens, K., McCarthy, I. P., \& Silvestre, B. S. (2011). Social media? Get serious! Understanding the functional building blocks of social media. Business Horizons, 54(3), 241-251.

[40] Kaplan RS, Norton DP: The Balanced Scorecard Measures that Drive Performance. Harvard Business Review 1992:71-79.

[41] Leung, Louis. "Generational differences in content generation in social media: The roles of the gratifications sought and of narcissism." Computers in Human Behavior 29.3 (2013): 997-1006. 